Selcuk Journal of Agriculture and Food Sciences

http://sjafs.selcuk.edu.tr/sjafs/index

Research Article
SJAFS

(2021) 35 (1), 24-32

e-ISSN: $2458-8377$

DOI:10.15316/SJAFS.2020.224

\title{
A New Approach on the Determination of the Spatial Criteria and Parameters Affecting Walking Comfort: Selcuk University Example***
}

\author{
(D) Ahmet AKAY ${ }^{1 *}$, (D) Serpil ÖNDER ${ }^{1}$ \\ ${ }^{1}$ Selçuk University, Faculty of Architecture and Design, Department of Landscape Architecture, Konya, Turkey
}

\begin{tabular}{l}
\hline ARTICLE INFO \\
\hline Article history: \\
Received date: 16.02 .2021 \\
Accepted date: 03.03 .2021 \\
\hline
\end{tabular}

Edited by:

Ahmet Tuğrul POLAT; Selcuk University, Turkey

Keywords:
Active Transport
Campus
Landscape Design
Open Space
Pedestrian
Walkability

Keywords:

Active Transport

Landscape Design

Walkability

\begin{abstract}
Physical inactivity and the diseases such as obesity are the results of increasing environmental pollution caused by developing technology, are shown as the main problems reducing the quality of life today. Succeed in the studies conducted on the topic of active transportation is directly related to the mentioned problems, which is very important in terms of reducing the negative effects. Although different methods are used in the relevant literature, a significant number of parameters are commonly evaluated in many studies. It is seen that these parameters are used in different study areas and there are inconsistencies between the results. Some control studies are needed to reveal the factors that cause inconsistent results. At this point, instead of using a standard set of parameters in various study areas, it would be useful to create a new set of specific parameters based on the characteristics of the related study area. This study aims to determine the spatial parameters affecting walking comfort in a specific study area (Selcuk University) together with the significance levels and to develop a new approach that can be adapted to different study areas based on this example area. In the study, both objective and subjective measurement methods were used to evaluate the potential inconsistency between the obtained results. In 17 of 30 parameters evaluated within the scope of the study, the coefficient determined by the users was higher than that of the experts. Based on the data that more than half of the parameters according to users are more important than those indicated by the experts, it was concluded that the standards and criteria were not fully accepted by the users.
\end{abstract}

\section{Introduction}

The use of motor vehicles (passive transportation), has increased as a result of the development of technology, causes a significant increase in greenhouse gas emissions. In Europe, 25.4\% of the $\mathrm{CO}_{2}$ emitted by passive transport is largely due to road transport (European Commission, 2014). With the increase of greenhouse gas emissions, air pollution also increases, especially in urban areas. This increase reflects the negative effects of passive transport on the environment and human health. The main source of the negative effects on human health is the fact that individuals start to adopt a more sedentary lifestyle due to the increase in the use of motor vehicles. At this point, the data that 3.2 million people die due to inactivity every year (WHO, 2010) indicates the significance of the issue.

\footnotetext{
* Corresponding author email: ahmetakay@selcuk.edu.tr

$* *$ This study has been produced from a part of the first author's doctorate thesis prepared under the supervision of the second author.
}

According to World Health Organization data, physical inactivity (lack of physical activity) has been identified as the fourth major risk factor among global causes of death (WHO, 2010). For this reason, accessible spaces should be created and individuals should be encouraged to use public transport and/or active modes of transport such as cycling and walking, which increase physical activity, instead of using personal motor vehicles (Davison, Ahern, \& Hine, 2015).

One of the most important factors that enable an area to function successfully in the context of being physically active is that the place has a high level of walkability or in other words, it has favorable conditions in terms of walking comfort. Several factors affect walking comfort, so the determination of the mentioned factors with their significance levels is essential to increase the level of walkability.

Research on urban design and walking generally focuses on macro-scale features of the physical environment, such as block length and the number of intersections, that can be measured remotely with the help of Geographical Information Systems (GIS) and aerial photographs. In contrast, urban designers emphasize 
the importance of micro-scale features for individuals to experience neighborhood environments ( $\mathrm{M}$. Alfonzo, Boarnet, Day, Mcmillan, \& Anderson, 2008). Besides, it was stated that there is a need for research on the real effects of micro-scale comfort elements such as urban design facilities (street furniture, fountains, etc.) (M. A. Alfonzo, 2005).

In the literature on the determination of parameters affecting the walking activity and walkability analysis, it is seen that approximately 25 different methods are used. Nyunt et al. (2015) stated that in previous studies (McGinn, Evenson, Herring, Huston, \& Rodriguez, 2007; Michael, Beard, Choi, Farquhar, \& Carlson, 2006), similar results could not be obtained regarding the objective and perceived (subjective) measures of the built environment, therefore, the same aspects of the built environment should be determined by both objective and subjective measurements. Considering the relevant literature in this context, while only objective measurement methods are used in some studies, subjective measurement methods are preferred in many studies, and in a small number of studies, it is seen that both measurement methods are used together. In the limited number of studies in which both objective and subjective measurement methods are used, it is understood that these 2 methods are not used as tools for comparison of the results, but as parts that contribute to reaching the entire (result).

By using the advanced search feature in the Web of Science database, scientific studies containing one of the terms "walkability", "walkable", "walk-friendly" and "walking comfort" in the title or keywords were searched and it was concluded that there were 1170 studies between the years of 2004-2020 (June). The notable diversity seen in the studies in terms of the research area is given in Figure 1. The fact that the subject is handled by researchers from different areas makes it possible to be shown as the main reason for the variety in the methods used in the related studies. As a result, the use of different methods causes inconsistent results. In this context, it seems that a new approach is needed for future researches on walking to reduce the inconsistencies in the results obtained by various methods used in the literature. The main features that should be found in the suggested new approach can be listed as follows:

- should be decisive about the boundaries of the research field and/or subject,

- $\quad$ should incorporate both objective and subjective measurement methods to increase the reliability of the results,

- should be easily adaptable for use in various working areas with different dynamics.

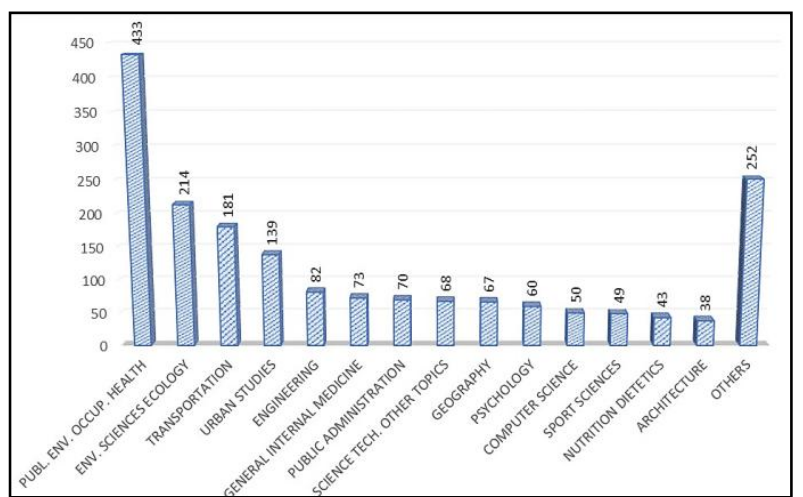

Figure 1

Distribution of studies conducted between 2004-2020 (June) by research areas

Based on the characteristics of the aforementioned approach, the following research questions were sought in this study:

1-) Are the spatial parameters frequently used in the literature about walking valid for every study area?

2-) How should spatial parameters affecting walking comfort be determined for a specific study area?

3-) Are the determining parameters equally significant for users and experts?

University campuses were chosen as the category of the sample study area, as they are places where young people are mostly together. The users of the Selcuk University Alaeddin Keykubat Campus contributed to the study during the subjective measurement process. The reason for the choice of university campuses as a category is that gaining the habit of walking at a young age increases the potential of individuals to continue these habits in the future. For this reason, the following goals were set within this study;

- Determination of spatial criteria and parameters that affect walking comfort in university campuses,

- Determination of the significance levels of the determined criteria and parameters by both expert and user opinions,

- Comparison and analysis of the results obtained by two different methods (objective and subjective)

The results of the study are expected to contribute significantly to the design processes of existing and future university campuses by providing qualified and necessary data to increase the comfort level of walking.

\section{Materials and Methods}

The possibility of developing innovative, comprehensive, and appropriate solutions as a result of any research is directly related to the elaboration of the study method and the participation of all relevant stakeholders in the process as much as possible. Therefore, in the process of preparing the method of this research, it was decided to include experts from various 
professions (architect, landscape architect, urban and regional planner, sociologist, psychologist, etc.) to the study in addition to the users of the Campus of Selçuk University. It was also decided to use both qualitative and quantitative research methods to test the obtained data and reach the most accurate results. To minimize the problems that may be encountered during the research, the study design was completed and the flow chart summarizing the study process was prepared (Figure 2).

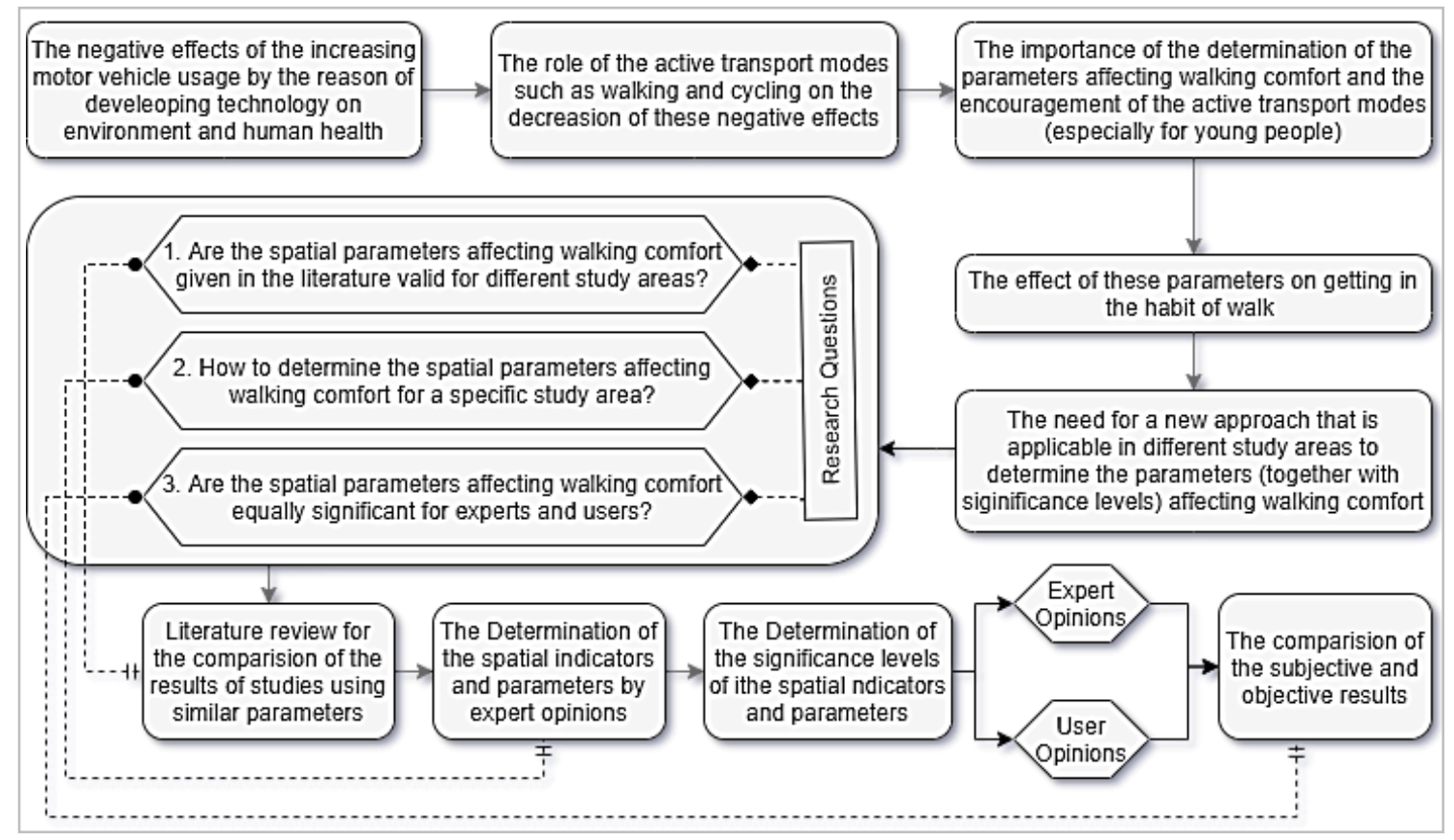

Figure 2

Study design and flow chart summarizing the process

Expert opinions were consulted to determine the criteria and parameters to be evaluated within the scope of this study. Before the expert opinions were consulted, the parameters that were frequently addressed in the relevant literature were determined. The determined parameters were grouped under categories and transformed into tables for the evaluation of experts, and the Delphi Technique was applied. This technique was developed in the 1950 s by two researchers, Olaf Helmer and Norman Dalkey, from the USA. The purposes of the Delphi Technique are; to make predictions about the future, to reveal expert opinions, and to reach consensus. Generally, the Delphi technique has three features:

(1) confidentiality in participation,

(2) statistical analysis of group response,

(3) controlled feedback.

The use of the Delphi technique usually includes sequential questionnaires applied to experts. Application results are shared with the participants after each application. This process continues until a consensus is reached. The achieved consensus is the product of this process (Şahin, 2001).

During the Delphi Technique implementation process, an online opinion was requested to reach a wide range of experts. The e-mail addresses of 273 faculty members from 41 universities from the Turkish Repub- lic of Northern Cyprus (K.K.T.C.) and Turkey, where "Landscape Architecture" and "Urban Design and Landscape Architecture" departments are located were obtained from the university web pages. The data repository table (criteria and parameters) created was sent to the addresses obtained and an evaluation was requested. The main reason why faculty members in the departments of Landscape Architecture are chosen as the target audience in determining the criteria and parameters is that the profession that performs open space design studies especially at the micro-level is Landscape Architecture and the subject of study is basically within the field of expertise of this profession.

After the process was completed in line with the opinions and suggestions of the experts, spatial criteria and parameters affecting walking comfort in university campuses were determined. In order to determine the significance (coefficients) of the criteria and parameters, two different (objective and subjective) evaluations were made by applying separate questionnaires to both experts and users based on the study of Maghelal and Capp (2011) (Figure 3). tists])

1-) Objective Evaluation (Expert opinions [Scien-

2-) Subjective Evaluation (User opinions [student, academic and administrative staff]) 


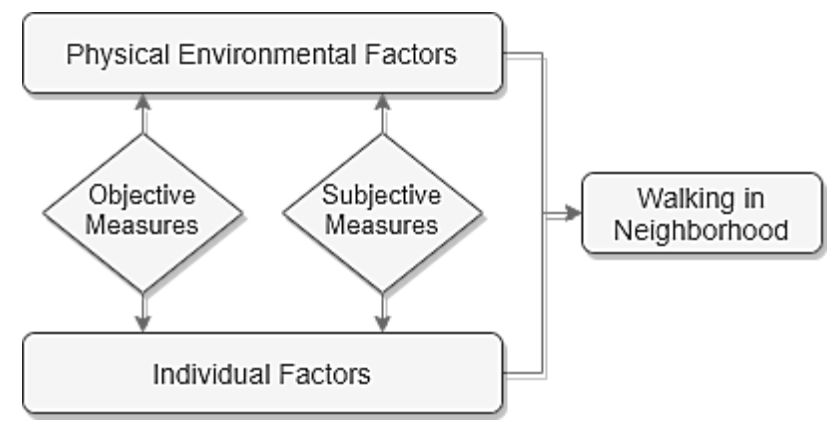

Figure 3

Factors affecting walkability and measurement methods (Maghelal \& Capp, 2011)

In both methods, a 9-point scoring system (from 1 to 9 increasing the significance level) was used to determine the significance of the parameters to be evaluated. The values and equivalents in this scoring are as follows:

1-Lowest importance, 3- Low importance,

5-Medium importance, 7-High importance,

9-Highest importance,

2,4,6,8- Intermediate values

As a result of the obtained scores, two different significance levels (both objective and subjective coefficients) of each parameter were obtained.

In the objective measurement phase where expert opinions were questioned, survey forms were prepared on "Google Forms", a professional online survey platform created for users to design surveys and collect data. The e-mail addresses of the academicians to whom the forms will be sent were also obtained from the university web pages. At this point, since different perspectives will greatly contribute to the objectivity of the study, opinions were taken from various professions (Landscape Architecture, Urban and Regional Planning, Architecture, Sociology, Civil Engineering). The scoring forms were sent to 872 academicians, 841 from Turkey, and 31 at the international level, by email. Responses were received from a total of 100 academicians, 95 at national and 5 at the international level. The following equation (Equation 1) was created, showing the mathematical expression of the operations performed in the calculation of the data.

Equation 1: Objective coefficient formula

$$
C_{o}=\prod_{i=1}^{n} S_{e i} \cdot S_{e c}
$$

$i=$ parameter number

$C_{o}=$ objective coefficient of the parameter

$S_{e}=$ significance score of the parameter according to experts

$S_{e c}=$ significance score of the relevant criteria according to experts

User scoring forms were prepared in the same format as the web-based scoring forms prepared for experts, to be used in the subjective measurement phase where user opinions are questioned. These forms were filled by the users on the campus by face-to-face interview method. The sample size calculations made for the survey application at this stage are based on the number of 65,000 people who regularly use the Selçuk University AlaeddinKeykubat Campus, reported by the university administration. Using the formula of Newbold (1995), the sample size was calculated as 166 with a $99 \%$ confidence interval and 0.1 error margin. The following equation (Equation 2) was created, showing the mathematical expression of the operations performed in the calculation of the data.

Equation 2: Subjective coefficient formula

$$
C_{s}=\prod_{i=1}^{n} S_{u i} \cdot S_{u c}
$$

$i=$ parameter number

$C_{s}=$ subjective coefficient of the parameter

$S_{u}=$ significance score of the parameter according to users

$S_{u c}=$ significance score of the relevant criteria according to users

The data obtained from both evaluation processes were compared, inconsistencies were determined and possible reasons for the differences between the results were addressed. As a result of all analyzes, the parameters that should be taken into account in the design processes of the walkable university campuses have been revealed with the data obtained.

\section{Results and Discussion}

\subsection{The Spatial Criteria and Parameters}

As a result of the feedback received from the academicians, the table containing the criteria and parameters was created (Table 1). A total of 30 spatial parameters were determined and these parameters were categorized under 4 criteria: Perceptual / Conceptual, Physical / Formal, Structural, and Vegetational. When the distribution of parameters is examined according to the criteria, it is seen that there are 6 spatial parameters under Perceptual/Conceptual criteria, 7 under Physical/Formal criteria, 11 under Structural criteria, and 6 under Vegetational criteria. Short codes shown in Table 1 were defined for the parameters by numbering together with the first 3 letters of the criteria category. Since the effects of the 4 criteria and 30 parameters determined with expert opinions on walking comfort are not the same, the significance levels should be determined before the measurements.

\subsection{Characteristics of the Expert Participants}

The faculty, department, and academic title information about the experts who participated in the study are given in Figure 4. It is seen that the highest participation in the survey based on faculties is from the faculties of architecture with 34 academic staff. According to academic titles, the highest participation was provid- 
ed from the Asst. Prof. Dr. titled academic staff. Based on departments, the highest participation was provided from Landscape Architecture with 45 academicians, Urban and Regional Planning with 23 academicians, and Architecture departments with 14 academicians.

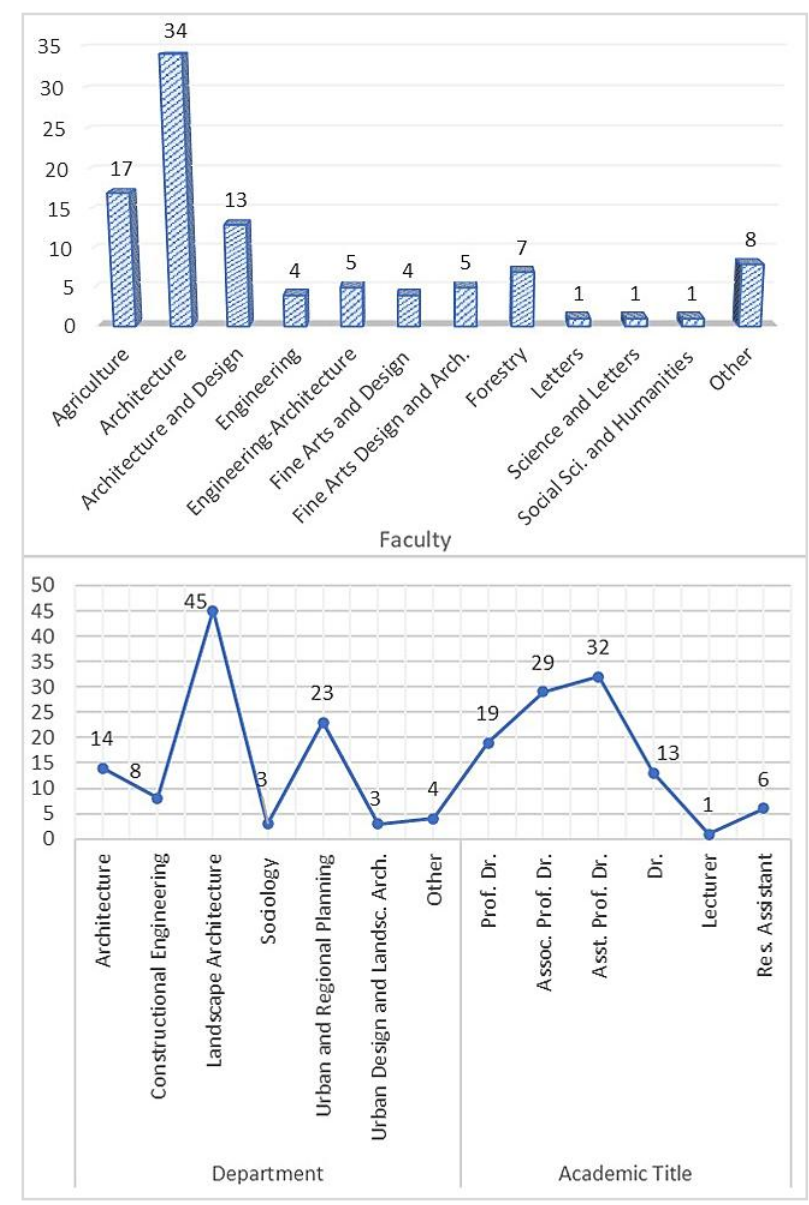

Figure 4

Faculty, department, and title information of the academicians who participated in the survey

\subsection{Characteristics of the User Participants}

The frequency analysis results of the demographic characteristics and the answers given by the users to the basic questions about and walking behaviors are shown in Table 2.

$56.6 \%$ of the 166 participants who answered the questionnaire are males. As expected, since the research is carried out on the university campus, $91 \%$ of the participants are students; $95.2 \%$ consists of young people group between the ages of $18-24.76 .5 \%$ of the participants answered "yes" and $22.3 \%$ of them answered "partially" to the question of "Do you like walking?". "Transit" response at a rate of $61.4 \%$ and "recreation" response at a rate of $34.3 \%$ were received to the question of "What is your reason for walking mostly?"

\subsection{Significance Levels (Coefficients) of the Spatial Criteria and Parameters}

The objective and subjective coefficients obtained as a result of normalizing the scores given by experts and users (so that the sum of the significance scores of all parameters is 100) calculated by using the objective and subjective coefficient formulas (see Equation 1 and Equation 2) are given in Table 1.

\subsubsection{Experts' Coefficients}

The number of scores given by the experts for the determination of significance levels of the spatial parameters affecting walking comfort on university campuses is shown in Table 3.

One of the perceptual/conceptual parameters, "pedestrian way occupation" was scored completely (9) by 52 experts. Following this parameter, "vehicle traffic density" got full points from 38 experts.

Among the physical / formal parameters, "continuity of pedestrian way" received a complete score (9) by 49 experts. The "slope" following this parameter received full points from 36 experts.

One of the structural parameters, "lighting", received a full score (9) by 36 experts. Following this parameter, "tactile floor covering material for sightdisabled people" received full points from 35 experts.

Among the vegetational parameters, "shadow trees" were scored completely (9) by 53 experts. Following this parameter, the "plant (green) buffer between pedestrian and motorway" received full points from 25 experts.

In order to increase the legibility of data obtained from experts, the highest number of scores given by experts for each parameter are given in bold format on Table 3.

It can be seen from Table 3 , the top 3 parameters that get the most 9 points from the experts are, "Shadow tree", "Pedestrian way occupation" and "Continuity of pedestrian way", respectively. The parameter that gets the most 1 point is "Drinking fountain".

As a result of the expert evaluations, it can be seen from Table 1 that the most significant parameter is "pedestrian way occupation" and the "continuity of pedestrian way" parameter, which has the closest coefficient to this parameter, is in the second place. It can also be seen that the lowest important parameter is "fountain".

\subsubsection{Users' Coefficients}

User scoring forms were prepared for campus users in the same format as the web-based forms prepared for experts. The data obtained with the surveys applied to 166 users on the campus were calculated with the subjective coefficient formula (see Equation 2) and the coefficients are given in Table 1. 
Table 1

The significance levels determined by experts' and users' opinions (objective and subjective coefficients)

\begin{tabular}{|c|c|c|c|c|}
\hline Criteria & Short Code & Parameter & Experts' Coefficient & $\begin{array}{c}\text { Users' } \\
\text { Coefficient }\end{array}$ \\
\hline \multirow{6}{*}{ 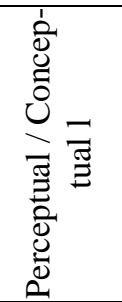 } & Per-1 & Pedestrian density & 3,7206 & 3,4307 \\
\hline & Per-2 & Vehicle traffic density & 3,8135 & 3,3221 \\
\hline & Per-3 & Noise & 3,5813 & 3,5538 \\
\hline & Per-4 & Smell & 3,5968 & 3,9229 \\
\hline & Per-5 & Visual quality & 3,6329 & 3,6840 \\
\hline & Per-6 & Pedestrian way occupation & 4,1748 & 4,3463 \\
\hline \multirow{7}{*}{ 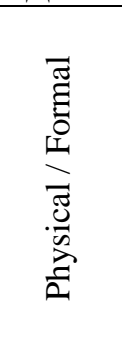 } & Phy-1 & Walkway shape (linear / curvilinear) & 2,6574 & 2,9148 \\
\hline & Phy-2 & Central refuge & 2,6777 & 2,7513 \\
\hline & Phy-3 & Slope & 3,6685 & 4,0174 \\
\hline & Phy-4 & Direction of pedestrian way (North-South etc.) & 2,9927 & 2,2713 \\
\hline & Phy-5 & Width of pedestrian way & 3,7650 & 3,5096 \\
\hline & Phy-6 & Continuity of pedestrian way & 4,0648 & 3,6000 \\
\hline & Phy-7 & Pedestrian crossing & 3,4602 & 3,3287 \\
\hline \multirow{11}{*}{ 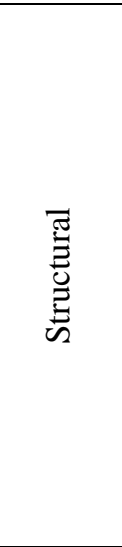 } & Str-1 & Floor covering material (ergonomics) & 3,5086 & 3,6348 \\
\hline & Str-2 & Tactile floor covering material for sight-disabled people & 3,6224 & 2,6818 \\
\hline & Str-3 & Curb ramp & 3,5680 & 2,9159 \\
\hline & Str-4 & Bollard / barrier (between pedestrian and vehicles) & 3,1820 & 2,9226 \\
\hline & Str-5 & Drainage condition & 3,5284 & 3,6816 \\
\hline & Str-6 & Bicycle road & 3,0583 & 2,6015 \\
\hline & Str-7 & Drinking fountain & 2,4348 & 2,4778 \\
\hline & Str-8 & Trash cans & 2,8653 & 2,9426 \\
\hline & Str-9 & Bench and seating area & 3,1820 & 3,2603 \\
\hline & Str-10 & Lighting & 3,7659 & 3,7485 \\
\hline & Str-11 & Maintenance / cleaning status of structural elements & 3,5581 & 3,4409 \\
\hline \multirow{6}{*}{ 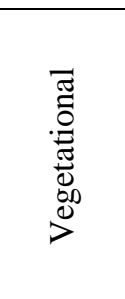 } & Veg-1 & Plant (green) buffer between pedestrian and motorway & 3,2002 & 3,6583 \\
\hline & Veg-2 & Shadow tree & 3,7551 & 4,2064 \\
\hline & Veg-3 & Shrub & 2,6175 & 3,0230 \\
\hline & Veg-4 & Flower & 2,6915 & 3,1522 \\
\hline & Veg-5 & Grass area & 2,6036 & 3,3197 \\
\hline & Veg-6 & Maintenance status of plants & 3,0522 & 3,6793 \\
\hline
\end{tabular}

Table 2

The frequency analysis results of the user participants

\begin{tabular}{llrrrr} 
Survey Question & Option & Frequency (n) & Percent (\%) & Valid Percent & Cumulative Percent \\
\hline Sex & Female & 72 & 43,4 & 43,4 & 43,4 \\
& Male & 94 & 56,6 & 56,6 & 100 \\
\hline Age & $18-24$ & 158 & 95,2 & 95,2 & 95,2 \\
& $25-34$ & 8 & 4,8 & 4,8 & 100 \\
\hline Participant Type & Student & 151 & 91 & 91 & 91 \\
& Administrative personal & 2 & 1,2 & 1,2 & 92,2 \\
& Worker & 5 & 3 & 3 & 95,2 \\
& Other & 8 & 4,8 & 4,8 & 100 \\
\hline
\end{tabular}


Table 2

The frequency analysis results of the user participants

\begin{tabular}{|c|c|c|c|c|c|}
\hline \multirow{4}{*}{ Education } & Master degree & 14 & 8,4 & 8,4 & 8,4 \\
\hline & Bachelor's degree & 130 & 78,3 & 78,3 & 86,7 \\
\hline & Two-year degree & 7 & 4,2 & 4,2 & 91 \\
\hline & Not Student & 15 & 9 & 9 & 100 \\
\hline \multirow[t]{5}{*}{ Monthly income (TL) } & $<2020$ & 151 & 91 & 91 & 91 \\
\hline & $2020-3500$ & 7 & 4,2 & 4,2 & 95,2 \\
\hline & $3501-4500$ & 4 & 2,4 & 2,4 & 97,6 \\
\hline & $4501-5500$ & 3 & 1,8 & 1,8 & 99,4 \\
\hline & $>5500$ & 1 & 0,6 & 0,6 & 100 \\
\hline \multirow[t]{3}{*}{ Do you like walking? } & No & 2 & 1,2 & 1,2 & 1,2 \\
\hline & Yes & 127 & 76,5 & 76,5 & 77,7 \\
\hline & Partially Yes & 37 & 22,3 & 22,3 & 100 \\
\hline \multirow[t]{3}{*}{ What is your reason for walking mostly? } & Transit & 102 & 61,4 & 61,4 & 61,4 \\
\hline & Sport & 7 & 4,2 & 4,2 & 65,7 \\
\hline & Recreation & 57 & 34,3 & 34,3 & 100 \\
\hline
\end{tabular}

Table 3

The number of scores given by the experts

\begin{tabular}{|c|c|c|c|c|c|c|c|c|c|}
\hline Score / Short Code & 1 Point & 2 Points & 3 Points & 4 Points & 5 Points & 6 Points & 7 Points & 8 Points & 9 Points \\
\hline Per-1 & 0 & 0 & 4 & 5 & 6 & 12 & 25 & 20 & 28 \\
\hline Per-2 & 2 & 1 & 1 & 4 & 9 & 5 & 21 & 19 & 38 \\
\hline Per-3 & 2 & 1 & 3 & 3 & 14 & 10 & 26 & 12 & 29 \\
\hline Per-4 & 0 & 4 & 4 & 4 & 10 & 12 & 20 & 15 & 31 \\
\hline Per-5 & 0 & 0 & 2 & 6 & 14 & 9 & 29 & 13 & 27 \\
\hline Per-6 & 0 & 0 & 0 & 3 & 2 & 7 & 11 & 25 & 52 \\
\hline Phy-1 & 2 & 8 & 18 & 11 & 15 & 12 & 20 & 6 & 8 \\
\hline Phy-2 & 1 & 6 & 17 & 12 & 19 & 14 & 16 & 11 & 4 \\
\hline Phy-3 & 1 & 1 & 6 & 4 & 6 & 8 & 21 & 17 & 36 \\
\hline Phy-4 & 2 & 6 & 11 & 5 & 18 & 9 & 26 & 11 & 12 \\
\hline Phy-5 & 0 & 0 & 2 & 3 & 7 & 10 & 24 & 26 & 28 \\
\hline Phy-6 & 0 & 0 & 0 & 3 & 2 & 6 & 19 & 21 & 49 \\
\hline Phy-7 & 2 & 1 & 4 & 2 & 11 & 14 & 29 & 18 & 19 \\
\hline Str-1 & 0 & 4 & 2 & 5 & 6 & 14 & 24 & 12 & 33 \\
\hline Str-2 & 1 & 2 & 3 & 1 & 10 & 6 & 23 & 19 & 35 \\
\hline Str-3 & 0 & 3 & 5 & 1 & 10 & 9 & 18 & 20 & 34 \\
\hline Str-4 & 0 & 4 & 4 & 3 & 23 & 19 & 14 & 13 & 20 \\
\hline Str-5 & 0 & 1 & 2 & 4 & 10 & 8 & 34 & 16 & 25 \\
\hline Str-6 & 2 & 3 & 6 & 4 & 18 & 16 & 28 & 13 & 10 \\
\hline Str-7 & 5 & 7 & 14 & 15 & 20 & 13 & 18 & 5 & 3 \\
\hline Str-8 & 1 & 4 & 8 & 11 & 20 & 15 & 24 & 9 & 8 \\
\hline Str-9 & 1 & 1 & 5 & 6 & 20 & 14 & 21 & 18 & 14 \\
\hline Str-10 & 0 & 1 & 0 & 1 & 6 & 12 & 23 & 21 & 36 \\
\hline Str-11 & 0 & 0 & 4 & 4 & 9 & 13 & 23 & 16 & 31 \\
\hline Veg-1 & 1 & 2 & 3 & 6 & 8 & 12 & 27 & 16 & 25 \\
\hline Veg-2 & 0 & 0 & 1 & 3 & 2 & 3 & 12 & 26 & 53 \\
\hline Veg-3 & 1 & 5 & 10 & 10 & 18 & 18 & 24 & 7 & 7 \\
\hline Veg-4 & 1 & 3 & 8 & 12 & 21 & 16 & 19 & 11 & 9 \\
\hline Veg-5 & 2 & 7 & 8 & 10 & 21 & 13 & 22 & 7 & 10 \\
\hline Veg-6 & 2 & 2 & 5 & 7 & 11 & 11 & 26 & 16 & 20 \\
\hline
\end{tabular}

As a result of user evaluations, it can be seen from Table 1 that the most important parameter (higher coefficient according to the experts') is "pedestrian way occupation" as in experts', and the "shadow tree" parameter has the closest coefficient is in the second place. The least important parameter is seen to be the "direction of the pedestrian way".
3.5. The Comparison of the Expert and User Coefficients

According to the objective coefficients determined by experts and subjective coefficients determined by users, it can be seen that the subjective coefficient is high in 17 parameters, while the objective coefficient is high in 13 parameters.

As can be seen in Figure 5, parameters that were found more important by the experts are as follows: 
$>$ Perceptual criteria,

- pedestrian density,

- vehicle traffic density,

- noise

$>$ Physical criteria,

- the direction of the pedestrian way,

- width of the pedestrian way,

- continuity of the pedestrian way,

- pedestrian crossing

Structural criteria,

- tactile floor covering material,

- curb ramp,

- bollard / barrier,

- bicycle road,

- lighting,

- maintenance/cleaning status of structural elements

The parameters that were found more important by the users are as follows:

$>$ Perceptual criteria,

- smell

- visual quality

- pedestrian way occupation (parking on the pedestrian road, etc.)

$>$ Physical criteria,

- central refuge

- slope

- the direction of pedestrian way

$>$ Structural criteria,

- floor covering material (ergonomics)

- drainage condition

- drinking fountain

- trash cans

- bench and seating area

$>$ Vegetational criteria,

- All the parameters in this group

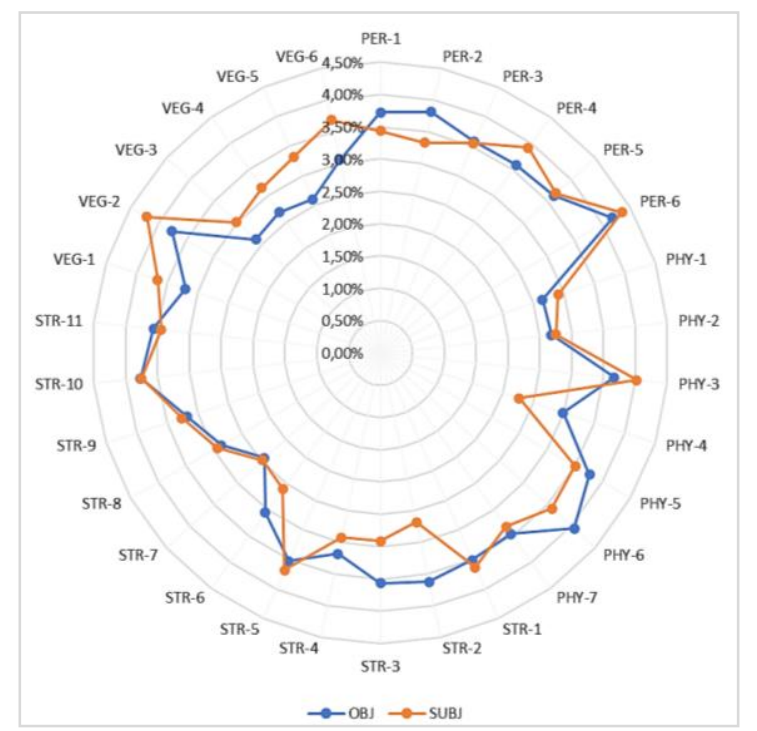

Figure 5

Radar chart of objective and subjective coefficients (significance levels)
Considering based on criteria, in 3 of the 4 criteria, several parameters are considered more important by both users and experts. Only all parameters under the vegetational criteria were found more important by users. Therefore, it is understood that the most significant difference in criteria basis is in vegetational criteria. When evaluated based on parameters, the user coefficient was found to be high in 17 out of 30 parameters. Based on the data that more than half of the parameters are of higher importance according to the users than those indicated by the experts, it has been concluded that the standards and criteria related to the mentioned parameters do not fully meet the users' needs.

As a result, answers were found in the processes of literature review and Delphi technique to research questions related to the determination of parameters and their validity. It was also understood at the stage when expert opinions were taken that the parameters frequently used in the literature on walking may not be valid in all areas. Based on the characteristics of the work area, the number of parameters may increase or decrease depending on the situation. Reaching the result with the feedback and exchange of ideas during the Delphi Technique process has shown that it is a very useful method to get opinions from professionals about the subject on the determination of the parameters. The method used in this study is, in a sense, an answer to the question of "How to determine the parameters affecting walking comfort for a specific area?". Finally, the data obtained in this study showed that the parameters are not equally important for experts and users. For this reason, it is important to organize the responsibilities and activity limits of both groups in the process of determining the parameters and significance levels. Experts need to take a more active role in the determination of the parameters that require more technical information. It is extremely important for the users, who are the owners of the work area in a sense, to take a more active role in the determination of the significance levels of the parameters determined in line by the technical knowledge and experiences of the experts.

\section{Conclusions}

Environmental pollution, inactivity, and the diseases they bring can be shown as the main indicators that summarize the most serious problems of our age and cause a decrease in the quality of life. Succeed in the studies carried out on the issue of active transportation, which has a direct effect on reducing the effects of the mentioned problems, is very important in this context.

The ongoing increase in the number of researches on active modes of transport is an important indicator that shows the seriousness of the issue. However, it is seen that the results obtained in most of the studies were conducted to contribute to the literature and the quality of life of the society, were not checked for validity. Therefore, it is understood that many studies 
have not achieved their goals. In this study, two different results were obtained with the inquiries made to different target groups in parallel with each other. As a result of the measurements, it was understood that some issues should be taken into consideration to reach the goals of the conducted studies.

The most important issues are:

- the determination of the purpose for walking

- the examination of the accuracy of the parameters to be evaluated and the significance levels in line with the determined walking purpose before the measurement process

Determination of the accuracy of the data is possible with the participation of the area users. The issue to be considered in user participation is that the objective measurement method used in the study should be adapted in a way that can be easily understood by the users. In many studies using both objective and subjective methods, it is seen that instead of the determination of the level of accuracy by the methods used, it is aimed to collect data for different characteristics of the field. The objective and subjective data obtained in these studies were used not to question the validity of the results, but as parts that complement each other and provide a single result. In some areas, it may not be possible to apply both methods. Because it is a difficult, expensive, and time-consuming application to get user opinion based on parameters. For this reason, in studies where only the objective measurement method is applied, users should at least be asked to give a score that shows their overall satisfaction with the field, even if there is no separate query based on parameters. Thus, it will be clarified whether the objective measurement results of the study are accepted by the users or not.

As a result, in terms of achieving successful results in researches related to active transportation, it is the most important point that experts and users are two significant parts of a whole. Besides, since each study area has its dynamics, factors determined according to the characteristics of the study area and users should be addressed rather than using a standard set of parameters generally accepted in the literature. In parallel with the success rate in the above-mentioned matters, the rate of contributing to the quality of life in social, cultural, economic, and ecological aspects, especially the environment and human health, will also increase.

\section{Acknowledgements}

This study has been produced from a part of the first author's doctorate thesis prepared under the supervision of the second author. This research was supported by a grant from the Coordinatorship of the Scientific Research Projects of Selçuk University (Project Number: 18101023).

\section{Declarations}

This research has the approval of the Scientific Research and Publication Ethics Committee of Selcuk University, with the decision numbered E.20083, dated 04/02/2021.

\section{References}

Alfonzo M, Boarnet MG, Day K., Mcmillan T, Anderson CL (2008). The relationship of neighbourhood built environment features and adult parents' walking. Journal of Urban Design, 13(1), 29-51.

Alfonzo MA (2005). To walk or not to walk? The hierarchy of walking needs. Environment and behavior, 37(6), 808-836.

Davison L, Ahern A, Hine J (2015). Travel, transport and energy implications of university-related student travel: A case study approach. Transportation Research Part D: Transport and Environment, 38, 27-40.

European Commission (2014). Transport Statistical Pocketbook 2014 http://ec.europa.eu/transport/factsfundings/statistics/doc/2014/pocketbook2014.pdf (accessed 15.02.2020).

Maghelal PK., Capp CJ (2011). Walkability: A Review of Existing Pedestrian Indices. Journal of the Urban \& Regional Information Systems Association, 23(2).

McGinn AP, Evenson KR, Herring AH, Huston SL, Rodriguez DA (2007). Exploring associations between physical activity and perceived and objective measures of the built environment. Journal of urban health, 84(2), 162-184.

Michael Y, Beard T, Choi D, Farquhar S, Carlson N (2006). Measuring the influence of built neighborhood environments on walking in older adults. Journal of aging and physical activity, 14(3), 302-312.

Nyunt MSZ, Shuvo FK., Eng JY, Yap KB, Scherer S, Hee LM, Ng TP (2015). Objective and subjective measures of neighborhood environment (NE): relationships with transportation physical activity among older persons. International journal of behavioral nutrition and physical activity, 12(1), 110.

Şahin AE (2001). Eğitim araştırmalarında delphi tekniği ve kullanımı. Hacettepe Üniversitesi Eğitim Fakültesi Dergisi, 20(20).

WHO (2010). Global recommendations on physical activity for health. World Health Organization. (ISBN:9789241599979) 